\title{
Existence of Positive Periodic Solutions for a Predator-Prey System of Holling Type IV Function Response with Mutual Interference and Impulsive Effects
}

\author{
Haidong Liu and Fanwei Meng \\ School of Mathematical Sciences, Qufu Normal University, Qufu 273165, China \\ Correspondence should be addressed to Haidong Liu; tomlhd983@163.com
}

Received 19 August 2014; Revised 17 January 2015; Accepted 18 January 2015

Academic Editor: Xue Z. He

Copyright (C) 2015 H. Liu and F. Meng. This is an open access article distributed under the Creative Commons Attribution License, which permits unrestricted use, distribution, and reproduction in any medium, provided the original work is properly cited.

We investigate the existence of periodic solutions for a predator-prey system with Holling function response and mutual interference. Our model is more general than others since it has both Holling type IV function and impulsive effects. With some new analytical tricks and the continuation theorem in coincidence degree theory proposed by Gaines and Mawhin, we obtain a set of sufficient conditions on the existence of positive periodic solutions for such a system. In addition, in the remark, we point out some minor errors which appeared in the proof of theorems in some published papers with relevant predator-prey models. An example is given to illustrate our results.

\section{Introduction}

In recent years, many authors [1-7] have extensively considered different types of predator-prey system. One of the typical systems is the following system:

$$
\begin{gathered}
\dot{x}(t)=x g(x)-\psi(x) y^{m}, \\
\dot{y}(t)=y\left(-d+k \psi(x) y^{m-1}-q(y)\right),
\end{gathered}
$$

which was introduced by Hassell in 1975 (see [8] for more details). The character of (1) is that it has the mutual interference constant $m(0<m<1)$. When Hassell studied the capturing behavior between the hosts (some bees) and parasite (a kind of butterfly), he noted that the hosts had the tendency to leave each other when they met, which interfered the hosts capturing effects. He also found that the mutual interference would be stronger while the populations of the parasite became larger and therefore he introduced the concept of mutual interference constant $m$. From then on, many authors began to study some kinds of predator-prey systems with mutual interference; see [9-12] for more details. Recently, Wang and Zhu [13] investigated a Volterra model with mutual interference and a Holling II type functional response

$$
\begin{gathered}
\dot{x}(t)=x(t)\left(r_{1}(t)-b_{1}(t) x(t)\right)-\frac{c_{1}(t) x(t)}{k^{2}+x(t)} y^{m}(t), \\
\dot{y}(t)=y(t)\left(-r_{2}(t)-b_{2}(t) y(t)\right)+\frac{c_{2}(t) x(t)}{k^{2}+x(t)} y^{m}(t) .
\end{gathered}
$$

And Wang et al. [14] discussed a Volterra model with mutual interference and a Holling III type functional response:

$$
\begin{gathered}
\dot{x}(t)=x(t)\left(r_{1}(t)-b_{1}(t) x(t)\right)-\frac{c_{1}(t) x^{2}(t)}{k^{2}+x^{2}(t)} y^{m}(t), \\
\dot{y}(t)=y(t)\left(-r_{2}(t)-b_{2}(t) y(t)\right)+\frac{c_{2}(t) x^{2}(t)}{k^{2}+x^{2}(t)} y^{m}(t),
\end{gathered}
$$

where

$$
\frac{x^{2}}{k^{2}+x^{2}}=p(x)
$$

is the Holling III type predation function. We can easily get $\dot{p}(x)>0$ for $x>0$ which shows that the predation 
rate increases with the increasing prey population density. But some experiments and observations indicate that the nonmonotonic response occurs at a level: when the nutrient concentration reaches a high level, an inhibitory effect on the specific growth rate may occur. That means that the predation function $p(x)$ may not always increase. To describe such inhibitory effect, Andrews in 1968 (see [15] for more details) suggested another type of Holling function called Holling type IV function

$$
p(x)=\frac{m x}{\alpha^{2}+\omega x+\beta x^{2}} .
$$

On the other hand, because of many natural and manmade factors, such as fire, drought, flooding, hunting, and harvesting, the intrinsic discipline of biological species usually undergoes some discrete changes of relatively short duration at some fixed times. More appropriate mathematical models for those situations are probably systems with impulsive effects. In recent years, many researchers have investigated several kinds of impulsive differential equations (see [16-27] and the references therein).

In this paper, we consider the following predator-prey system of Holling type IV function response with mutual interference and impulsive effects:

$$
\begin{gathered}
\dot{x}(t)=x(t)\left(r_{1}(t)-b_{1}(t) x(t)\right) \\
-\frac{c_{1}(t) x(t)}{\alpha^{2}+\omega x(t)+\beta x^{2}(t)} y^{m}(t), \\
\dot{y}(t)=y(t)\left(-r_{2}(t)-b_{2}(t) y(t)\right) \\
+\frac{c_{2}(t) x(t)}{\alpha^{2}+\omega x(t)+\beta x^{2}(t)} y^{m}(t), \\
\Delta x\left(t_{k}\right)=x\left(t_{k}^{+}\right)-x\left(t_{k}\right)=d_{1 k} x\left(t_{k}\right), \quad k=1,2, \ldots, \\
\Delta y\left(t_{k}\right)=y\left(t_{k}^{+}\right)-y\left(t_{k}\right)=d_{2 k} y\left(t_{k}\right), \quad k=1,2, \ldots,
\end{gathered}
$$

where $x(t)$ denotes the density of the prey population and $y(t)$ denotes the density of the predator population; $r_{1}(t)$ is the growth rate of the prey in the absence of predator; $r_{2}(t)$ is the death rate of predator in the absence of prey; $b_{1}(t)$ is the decay rate of the prey in the competition among the preys; $b_{2}(t)$ is the decay rate of the predator in the competition among the predators; $c_{1}(t)$ is the predation rate of predator, and $c_{2}(t)$ is the coefficient of transformation from preys to predators; $d_{1 k} x\left(t_{k}\right)$ and $d_{2 k} y\left(t_{k}\right)$ represent the populations $x(t)$ and $y(t)$ at $t_{k}$ regular harvest pulse.

By use of the continuation theorem in coincidence degree theory and some new analytical tricks, we have derived sufficient conditions for the existence of positive periodic solutions of the general system (6). In proving the theorem, we have avoided the errors that exist in the existing articles. We also provide an example to illustrate our theorem.

\section{Preliminaries}

Definition 1. A function $z(t)=(x(t), y(t))^{\top} \in \mathrm{R}^{2}$ is said to be a $T$-periodic solution of system (6), if it satisfies the following conditions:

(i) $z(t)$ is a piecewise continuous map with first-class discontinuity points in $\left\{t_{k}\right\} \cap[0, T]$, and each discontinuity point is continuous on the left,

(ii) $z(t)$ satisfies system (6) in the interval $[0, T]$,

(iii) $z(t)$ satisfies $z(t+T)=z(t), t \in[0, \infty)$.

Throughout this paper, the following assumptions hold.

$\left[A_{1}\right] r_{1}(t), b_{1}(t), r_{2}(t), b_{2}(t), c_{1}(t)$, and $c_{2}(t)$ are all continuous positive periodic functions with a common period $T>0$.

$$
\begin{aligned}
{\left[A_{2}\right] } & 0<t_{1}<\cdots<t_{k}<t_{k+1}<\cdots \text { and } \lim _{k \rightarrow \infty} t_{k}= \\
& +\infty, t_{k} \neq T(k=1,2, \ldots), \alpha, \omega, \beta \text { are positive } \\
& \text { constants, } m \in(0,1), \text { and there exists a positive } \\
& \text { integer } q \text {, such that } t_{k+q}=t_{k}+T, d_{i(k+q)}=d_{i k} \in \\
& (-1,0](i=1,2),[0, T] \cap\left\{t_{k}\right\}=\left\{t_{1}, t_{2}, \ldots t_{q}\right\} \text { for } \\
& k=1,2, \ldots .
\end{aligned}
$$

Let $X$ and $Y$ be two Banach spaces, $L:$ Dom $L \subset X \rightarrow Y$ is a linear map, and $N: X \rightarrow Y$ is a continuous map. If $\operatorname{dim}$ $\operatorname{Ker} L=\operatorname{codim} \operatorname{Im} L<+\infty$ and $\operatorname{Im} L \subset Y$ are closed, then we call operator $L$ a Fredholm operator with index zero [28]. If $L$ is a Fredholm operator with index zero and there exist continuous projects $P: X \rightarrow X$ and $Q: Y \rightarrow Y$ such that $\operatorname{Im} P=\operatorname{Ker} L, \operatorname{Im} L=\operatorname{Ker} Q=\operatorname{Im}(I-Q)$, then $\left.L\right|_{\text {Dom } L \cap K e r P}:$ $(I-P) X \rightarrow \operatorname{Im} L$ has an inverse function, which we set as $K_{p}$. Assume $\Omega \subset X$ is any open bounded set, if $Q N(\bar{\Omega})$ is bounded and $K_{p}(I-Q) N(\bar{\Omega}) \subset X$ is relatively compact, then we say $N$ is $L$-compact on $\bar{\Omega}$. Since $\operatorname{Im} Q$ is isomorphic to $\operatorname{Ker} L$, there exists isomorphism $J: \operatorname{ImQ} \rightarrow \operatorname{Ker} L$. Now we come to the continuation theorem [28, page 40].

Lemma 2 (see [28], Continuation Theorem). Let $X$ and $Y$ be both Banach spaces, let $L: \operatorname{Dom} L \subset X \rightarrow Y$ be a Fredholm operator with index zero, let $\Omega \subset X$ be an open bounded set, and let $N: \bar{\Omega} \rightarrow Y$ be L-compact on $\bar{\Omega}$. If all the following conditions hold:

$$
\begin{aligned}
& {\left[C_{1}\right] L x \neq \lambda N x \text { for each } \lambda \in(0,1), x \in \partial \Omega \cap \operatorname{Dom} L,} \\
& {\left[C_{2}\right] \text { for each } x \in \partial \Omega \cap \operatorname{Ker} L, Q N x \neq 0,} \\
& {\left[C_{3}\right] \operatorname{deg}\{J Q N, \Omega \cap \operatorname{Ker} L, 0\} \neq 0,}
\end{aligned}
$$

then the equation $L x=N x$ has at least one solution on $\bar{\Omega} \cap$ Dom $L$. 
For the convenience, we denote

$$
\begin{gathered}
P C(\mathrm{R}, \mathrm{R})=\{\psi: \mathrm{R} \rightarrow \mathrm{R} \mid \psi \text { is continuous at } \\
t \neq t_{k}, \psi\left(t_{k}^{+}\right), \psi\left(t_{k}^{-}\right) \text {exist, } \\
\left.\psi\left(t_{k}\right)=\psi\left(t_{k}^{-}\right), k=1,2, \ldots\right\},
\end{gathered}
$$

$\widetilde{C}(R, R):=\{\psi: R \rightarrow R \mid \psi$ is continuous at

$$
\begin{gathered}
\left.t \neq t_{k}, \psi\left(t_{k}^{+}\right), \psi\left(t_{k}^{-}\right) \text {exist, } k=1,2, \ldots\right\} \\
\bar{f}=\frac{1}{T} \int_{0}^{T} f(t) d t, \quad f^{L}=\min _{t \in[0, T]} f(t), \\
f^{M}=\max _{t \in[0, T]} f(t) .
\end{gathered}
$$

\section{Existence of Positive Periodic Solutions}

Theorem 3. Besides $\left(A_{1}\right)$ and $\left(A_{2}\right)$, if there hold the following conditions:

$$
\bar{r}_{1} \geqslant \max \left\{-\frac{1}{T} \sum_{k=1}^{q} \ln \left(1+d_{1 k}\right),-\sum_{k=1}^{q} \ln \left(1+d_{1 k}\right)\right\},
$$

then system (6) has at least one positive T-periodic solution.

Proof. Suppose $(x(t), y(t)) \in \mathrm{R}^{2}$ is an arbitrary positive solution of system $(6)$. have

Let $x(t)=e^{u(t)}, y(t)=e^{v(t)}$, it follows from (6) that we

$$
\begin{aligned}
\dot{u}(t)= & r_{1}(t)-b_{1}(t) e^{u(t)} \\
& -\frac{c_{1}(t)}{\alpha^{2}+\omega e^{u(t)}+\beta e^{2 u(t)}} e^{m v(t)}, \\
\dot{v}(t)=- & r_{2}(t)-b_{2}(t) e^{v(t)} \\
& +\frac{c_{2}(t) e^{u(t)}}{\alpha^{2}+\omega e^{u(t)}+\beta e^{2 u(t)}} e^{(m-1) v(t),} \\
\Delta u\left(t_{k}\right) & =\ln \left(1+d_{1 k}\right), \quad k=1,2, \ldots, \\
\Delta v\left(t_{k}\right) & =\ln \left(1+d_{2 k}\right), \quad k=1,2, \ldots,
\end{aligned}
$$

It is easy to see that if system (9) has one $T$ periodic solution $\left(u^{*}(t), v^{*}(t)\right)^{\top}$, then $\left(x^{*}(t), y^{*}(t)\right)^{\top}=$ $\left(\exp \left[u^{*}(t)\right], \exp \left[v^{*}(t)\right]\right)^{\top}$ is a positive $T$-periodic solution of (6). Therefore, we need only to prove that (9) has one $T$-periodic solution.

To apply Lemma 2, we take

$$
\begin{gathered}
X=\left\{z(t)=(u(t), v(t))^{\top} \mid u(t) \in P C(\mathrm{R}, \mathrm{R}),\right. \\
v(t) \in P C(\mathrm{R}, \mathrm{R}), z(t+\mathrm{T})=z(t)\}
\end{gathered}
$$

with the norm

$$
\|z(t)\|_{X}=\left\|(u(t), v(t))^{\top}\right\|_{X}=\sup _{t \in[0, T]}|u(t)|+\sup _{t \in[0, T]}|v(t)|,
$$

and let

$$
\begin{array}{r}
\widetilde{X}=\left\{z(t)=(u(t), v(t))^{\top} \mid u(t) \in \widetilde{C}(\mathrm{R}, \mathrm{R}),\right. \\
v(t) \in \widetilde{C}(\mathrm{R}, \mathrm{R}), z(t+T)=z(t) \text { for } \\
\left.t \neq t_{k}, k=1,2, \ldots\right\}
\end{array}
$$

with the norm

$$
\begin{aligned}
\|z(t)\|_{\widetilde{X}} & =\left\|(u(t), v(t))^{\top}\right\|_{\widetilde{X}} \\
& =\sup _{t \in[0, T] \backslash\left\{t_{1}, t_{2}, \ldots, t_{q}\right\}}|u(t)|+\sup _{t \in[0, T] \backslash\left\{t_{1}, t_{2}, \ldots, t_{q}\right\}}|v(t)|, \\
Y & =\widetilde{X} \times \overbrace{\mathrm{R}^{2} \times \mathrm{R}^{2} \times \cdots \times \mathrm{R}^{2}}^{q}=\widetilde{X} \times \mathrm{R}^{2 q}
\end{aligned}
$$

be equipped with the norm

$$
\|\phi\|_{Y}=\|z\|_{\widetilde{X}}+\sum_{i=1}^{q}\left|v_{i}\right|_{2}, \quad \text { for } \phi=\left(z, v_{1}, \ldots, v_{q}\right) \in Y,
$$

where $|\cdot|_{2}$ denotes the Euclidean norm of $\mathrm{R}^{2}$. Then $\left(X,\|\cdot\|_{X}\right)$ and $\left(Y,\|\cdot\|_{Y}\right)$ are both Banach spaces.

Let

$$
\operatorname{Dom} L=\left\{z(t) \in X \mid \text { for } t \neq t_{k}, \dot{z}(t) \in \widetilde{X}\right\} \subset X,
$$

and define operators $L$ and $N$ as follows, respectively:

$$
\begin{aligned}
L(z(t)) & =L\left(\begin{array}{l}
u(t) \\
v(t)
\end{array}\right) \\
& =\left(\left(\begin{array}{ll}
\dot{u}(t), & \text { for } t \neq t_{k} \\
\dot{v}(t), & \text { for } t \neq t_{k}
\end{array}\right),\left\{\left(\begin{array}{c}
\Delta u\left(t_{k}\right) \\
\Delta v\left(t_{k}\right)
\end{array}\right)\right\}_{k=1}^{q}\right),
\end{aligned}
$$

$N(z(t))$

$$
=N\left(\begin{array}{l}
u(t) \\
v(t)
\end{array}\right)
$$

$=\left(\left(\begin{array}{c}r_{1}(t)-b_{1}(t) e^{u(t)}-\frac{c_{1}(t)}{\alpha^{2}+\omega e^{u(t)}+\beta e^{2 u(t)}} e^{m v(t)} \\ -r_{2}(t)-b_{2}(t) e^{v(t)}+\frac{c_{2}(t) e^{u(t)}}{\alpha^{2}+\omega e^{u(t)}+\beta e^{2 u(t)}} e^{(m-1) v(t)}\end{array}\right)\right.$,

$$
\left.\left\{\left(\begin{array}{l}
\ln \left(1+d_{1 k}\right) \\
\ln \left(1+d_{2 k}\right)
\end{array}\right)\right\}_{k=1}^{q}\right)
$$


then

$$
\operatorname{Ker} L=\mathrm{R}^{2}
$$$$
\operatorname{Im} L=\left\{y(t)=\left(\left(\begin{array}{l}
f(t) \\
g(t)
\end{array}\right),\left\{\left(\begin{array}{l}
a_{k} \\
e_{k}
\end{array}\right)\right\}_{k=1}^{q}\right)\right.
$$

$$
\left.\in Y \mid\left(\begin{array}{c}
\int_{0}^{T} f(t) d t+\sum_{k=1}^{q} a_{k}=0 \\
\int_{0}^{T} g(t) d t+\sum_{k=1}^{q} e_{k}=0
\end{array}\right)\right\}
$$

is closed in $Y$, and

$$
\operatorname{dim} \operatorname{Ker} L=2=\operatorname{codim} \operatorname{Im} L .
$$

It follows that $L$ is a Fredholm mapping of index zero, and it is easy to know that $P$ and $Q$ are both continuous projectors such that

$$
\operatorname{Im} P=\operatorname{Ker} L, \quad \operatorname{Im} L=\operatorname{Ker} Q=\operatorname{Im}(I-Q),
$$

where $P$ and $Q$ are defined by

$$
P\left(\begin{array}{l}
u(t) \\
v(t)
\end{array}\right)=\frac{1}{T}\left(\begin{array}{c}
\int_{0}^{T} u(t) d t+\sum_{k=1}^{q} a_{k} \\
\int_{0}^{T} v(t) d t+\sum_{k=1}^{q} e_{k}
\end{array}\right)
$$

where $\left\{\left(\begin{array}{c}a_{k} \\ e_{k}\end{array}\right)\right\}_{k=1}^{q}$ are arbitrary constant vector groups, if $\left(\begin{array}{c}u(t) \\ v(t)\end{array}\right) \in X \backslash \operatorname{Dom} L$, and $\left\{\left(\begin{array}{c}a_{k} \\ e_{k}\end{array}\right)\right\}_{k=1}^{q}$ satisfy

$$
\int_{0}^{t_{1}} \dot{u}(t) d t+\sum_{k=2}^{k=q} \int_{t_{k-1}}^{t_{k}} \dot{u}(t) d t+\int_{t_{q}}^{T} \dot{u}(t) d t+\sum_{k=1}^{q} a_{k}=0
$$

$$
\begin{aligned}
& \int_{0}^{t_{1}} \dot{v}(t) d t+\sum_{k=2}^{k=q} \int_{t_{k-1}}^{t_{k}} \dot{v}(t) d t+\int_{t_{q}}^{T} \dot{v}(t) d t+\sum_{k=1}^{q} e_{k}=0, \\
& \text { if }\left(\begin{array}{l}
u(t) \\
v(t)
\end{array}\right) \in \operatorname{Dom} L, \\
& Q\left(\left(\begin{array}{l}
u(t) \\
v(t)
\end{array}\right),\left\{\left(\begin{array}{l}
a_{k} \\
e_{k}
\end{array}\right)\right\}_{k=1}^{q}\right) \\
& =\left(\frac{1}{T}\left(\begin{array}{c}
\int_{0}^{T} u(t) d t+\sum_{k=1}^{q} a_{k} \\
\int_{0}^{T} v(t) d t+\sum_{k=1}^{q} e_{k}
\end{array}\right),\left\{\left(\begin{array}{l}
0 \\
0
\end{array}\right)\right\}_{k=1}^{q}\right) \text {, where } \\
& \left(\left(\begin{array}{l}
u(t) \\
v(t)
\end{array}\right),\left\{\left(\begin{array}{l}
a_{k} \\
e_{k}
\end{array}\right)\right\}_{k=1}^{q}\right) \in Y .
\end{aligned}
$$

From $L$ is a Fredholm operator with index zero, we get that $L$ has a unique inverse. We define $K_{p}: \operatorname{Im} L \rightarrow \operatorname{Ker} P \cap \operatorname{Dom} L$ as the generalized inverse to $L$, that is,

$$
\begin{aligned}
& K_{p}\left(\left(\begin{array}{l}
u(t) \\
v(t)
\end{array}\right),\left\{\left(\begin{array}{l}
a_{k} \\
e_{k}
\end{array}\right)\right\}_{k=1}^{q}\right) \\
& =\left(\begin{array}{c}
\int_{0}^{t} u(s) d s+\sum_{0<t_{k}<t} a_{k}-\frac{1}{T} \int_{0}^{T} \int_{0}^{t} u(s) d s d t-\sum_{k=1}^{q} a_{k} \\
\int_{0}^{t} v(s) d s+\sum_{0<t_{k}<t} e_{k}-\frac{1}{T} \int_{0}^{T} \int_{0}^{t} v(s) d s d t-\sum_{k=1}^{q} e_{k}
\end{array}\right) .
\end{aligned}
$$

$$
\begin{aligned}
& \mathrm{QN}\left(\begin{array}{l}
u(t) \\
v(t)
\end{array}\right) \\
& =\left(\begin{array}{c}
\frac{1}{T} \int_{0}^{T}\left(r_{1}(t)-b_{1}(t) e^{u(t)}-\frac{c_{1}(t)}{\alpha^{2}+\omega e^{u(t)}+\beta e^{2 u(t)}} e^{m v(t)}\right) d t+\frac{1}{T} \sum_{k=1}^{q} \ln \left(1+d_{1 k}\right) \\
\frac{1}{T} \int_{0}^{T}\left(-r_{2}(t)-b_{2}(t) e^{v(t)}+\frac{c_{2}(t) e^{u(t)}}{\alpha^{2}+\omega e^{u(t)}+\beta e^{2 u(t)}} e^{(m-1) v(t)}\right) d t+\frac{1}{T} \sum_{k=1}^{q} \ln \left(1+d_{2 k}\right)
\end{array}\right),\left\{\left(\begin{array}{l}
0 \\
0
\end{array}\right)\right\}_{k=1}^{q}, \\
& K_{p}(I-Q) N\left(\begin{array}{l}
u(t) \\
v(t)
\end{array}\right) \\
& =\left(\begin{array}{c}
\int_{0}^{t}\left(r_{1}(s)-b_{1}(s) e^{u(s)}-\frac{c_{1}(s)}{\alpha^{2}+\omega e^{u(s)}+\beta e^{2 u(s)}} e^{m v(s)}\right) d s+\sum_{0<t_{k}<t}^{q} \ln \left(1+d_{1 k}\right) \\
\int_{0}^{t}\left(-r_{2}(s)-b_{2}(s) e^{v(s)}+\frac{c_{2}(s) e^{u(s)}}{\alpha^{2}+\omega e^{u(s)}+\beta e^{2 u(s)}} e^{(m-1) v(s)}\right) d s+\sum_{0<t_{k}<t}^{q} \ln \left(1+d_{2 k}\right)
\end{array}\right)
\end{aligned}
$$




$$
\begin{aligned}
& -\left(\begin{array}{c}
\frac{1}{T} \int_{0}^{T} \int_{0}^{t}\left(r_{1}(s)-b_{1}(s) e^{u(s)}-\frac{c_{1}(s)}{\alpha^{2}+\omega e^{u(s)}+\beta e^{2 u(s)}} e^{m v(s)}\right) d s d t+\sum_{k=1}^{q} \ln \left(1+d_{1 k}\right) \\
\frac{1}{T} \int_{0}^{T} \int_{0}^{t}\left(-r_{2}(s)-b_{2}(s) e^{v(s)}+\frac{c_{2}(s) e^{u(s)}}{\alpha^{2}+\omega e^{u(s)}+\beta e^{2 u(s)}} e^{(m-1) v(s)}\right) d s d t+\sum_{k=1}^{q} \ln \left(1+d_{2 k}\right)
\end{array}\right) \\
& -\left(\begin{array}{c}
\frac{1}{T} \int_{0}^{t} \int_{0}^{T}\left(r_{1}(s)-b_{1}(s) e^{u(s)}-\frac{c_{1}(s)}{\alpha^{2}+\omega e^{u(s)}+\beta e^{2 u(s)}} e^{m v(s)}\right) d s d s \\
\frac{1}{T} \int_{0}^{t} \int_{0}^{T}\left(-r_{2}(s)-b_{2}(s) e^{v(s)}+\frac{c_{2}(s) e^{u(s)}}{\alpha^{2}+\omega e^{u(s)}+\beta e^{2 u(s)}} e^{(m-1) v(s)}\right) d s d s
\end{array}\right) \\
& -\frac{t}{T}\left(\begin{array}{c}
\sum_{k=1}^{q} \ln \left(1+d_{1 k}\right) \\
\sum_{k=1}^{q} \ln \left(1+d_{2 k}\right)
\end{array}\right) \\
& +\left(\begin{array}{c}
\frac{1}{T^{2}} \int_{0}^{T} \int_{0}^{t} \int_{0}^{T}\left(r_{1}(s)-b_{1}(s) e^{u(s)}-\frac{c_{1}(s)}{\alpha^{2}+\omega e^{u(s)}+\beta e^{2 u(s)}} e^{m v(s)}\right) d s d s d t \\
\frac{1}{T^{2}} \int_{0}^{T} \int_{0}^{t} \int_{0}^{T}\left(-r_{2}(s)-b_{2}(s) e^{v(s)}+\frac{c_{2}(s) e^{u(s)}}{\alpha^{2}+\omega e^{u(s)}+\beta e^{2 u(s)}} e^{(m-1) v(s)}\right) d s d s d t
\end{array}\right) \\
& +\frac{1}{2}\left(\begin{array}{c}
\sum_{k=1}^{q} \ln \left(1+d_{1 k}\right) \\
\sum_{k=1}^{q} \ln \left(1+d_{2 k}\right)
\end{array}\right) \\
& =\left(\begin{array}{c}
\int_{0}^{t}\left(r_{1}(s)-b_{1}(s) e^{u(s)}-\frac{c_{1}(s)}{\alpha^{2}+\omega e^{u(s)}+\beta e^{2 u(s)}} e^{m v(s)}\right) d s+\sum_{0<t_{k}<t}^{q} \ln \left(1+d_{1 k}\right) \\
\int_{0}^{t}\left(-r_{2}(s)-b_{2}(s) e^{v(s)}+\frac{c_{2}(s) e^{u(s)}}{\alpha^{2}+\omega e^{u(s)}+\beta e^{2 u(s)}} e^{(m-1) v(s)}\right) d s+\sum_{0<t_{k}<t}^{q} \ln \left(1+d_{2 k}\right)
\end{array}\right) \\
& -\left(\begin{array}{c}
\frac{1}{T} \int_{0}^{T} \int_{0}^{t}\left(r_{1}(s)-b_{1}(s) e^{u(s)}-\frac{c_{1}(s)}{\alpha^{2}+\omega e^{u(s)}+\beta e^{2 u(s)}} e^{m v(s)}\right) d s d t+\sum_{k=1}^{q} \ln \left(1+d_{1 k}\right) \\
\frac{1}{T} \int_{0}^{T} \int_{0}^{t}\left(-r_{2}(s)-b_{2}(s) e^{v(s)}+\frac{c_{2}(s) e^{u(s)}}{\alpha^{2}+\omega e^{u(s)}+\beta e^{2 u(s)}} e^{(m-1) v(s)}\right) d s d t+\sum_{k=1}^{q} \ln \left(1+d_{2 k}\right)
\end{array}\right) \\
& +\left(\frac{1}{2}-\frac{t}{T}\right)\left(\begin{array}{c}
\int_{0}^{T}\left(r_{1}(s)-b_{1}(s) e^{u(s)}-\frac{c_{1}(s)}{\alpha^{2}+\omega e^{u(s)}+\beta e^{2 u(s)}} e^{m v(s)}\right) d s \\
\int_{0}^{T}\left(-r_{2}(s)-b_{2}(s) e^{v(s)}+\frac{c_{2}(s) e^{u(s)}}{\alpha^{2}+\omega e^{u(s)}+\beta e^{2 u(s)}} e^{(m-1) v(s)}\right) d s
\end{array}\right) \\
& +\left(\frac{1}{2}-\frac{t}{T}\right)\left(\begin{array}{c}
\sum_{k=1}^{q} \ln \left(1+d_{1 k}\right) \\
\sum_{k=1}^{q} \ln \left(1+d_{2 k}\right)
\end{array}\right)
\end{aligned}
$$

By the Lebesqgue convergence theorem, QN and $K_{p}(I-Q) N$ are both continuous. From the Arzela-Ascoli Theorem, we can get that $K_{p}(I-Q) N(\bar{\Omega})$ is relatively compact and $Q N(\bar{\Omega})$ is bounded for any open set $\Omega \subset X$. So $N$ is $L$-compact on $\bar{\Omega}$ for any open bounded set $\Omega$. 
Now we consider the operator equation $L z=\lambda N z, \lambda \in$ $(0,1)$, that is,

$$
\begin{gathered}
\dot{u}(t)=\lambda\left(r_{1}(t)-b_{1}(t) e^{u(t)}-\frac{c_{1}(t)}{\alpha^{2}+\omega e^{u(t)}+\beta e^{2 u(t)}} e^{m v(t)}\right), \\
\dot{v}(t) \\
=\lambda\left(-r_{2}(t)-b_{2}(t) e^{v(t)}+\frac{c_{2}(t) e^{u(t)}}{\alpha^{2}+\omega e^{u(t)}+\beta e^{2 u(t)}} e^{(m-1) v(t)}\right), \\
t \neq t_{k}, \quad k=1,2, \ldots, \\
\Delta u\left(t_{k}\right)=\lambda \ln \left(1+d_{1 k}\right), \quad k=1,2, \ldots, \\
\Delta v\left(t_{k}\right)=\lambda \ln \left(1+d_{2 k}\right), \quad k=1,2, \ldots .
\end{gathered}
$$

Integrating (24) over the interval $[0, T]$ leads to

$$
\begin{aligned}
\int_{0}^{T} b_{1}(t) e^{u(t)} d t= & \int_{0}^{T} r_{1}(t) d t \\
& -\int_{0}^{T} \frac{c_{1}(t)}{\alpha^{2}+\omega e^{u(t)}+\beta e^{2 u(t)}} e^{m v(t)} d t \\
& +\sum_{k=1}^{q} \ln \left(1+d_{1 k}\right) \\
\int_{0}^{T} b_{2}(t) e^{v(t)} d t= & -\int_{0}^{T} r_{2}(t) d t \\
& +\int_{0}^{T} \frac{c_{2}(t) e^{u(t)}}{\alpha^{2}+\omega e^{u(t)}+\beta e^{2 u(t)}} e^{(m-1) v(t)} d t \\
& +\sum_{k=1}^{q} \ln \left(1+d_{2 k}\right) .
\end{aligned}
$$

From the first equation of (25), we have

$$
b_{1}^{L} \int_{0}^{T} e^{u(t)} d t \leqslant \int_{0}^{T} b_{1}(t) e^{u(t)} d t \leqslant \int_{0}^{T} r_{1}(t) d t=T \cdot \bar{r}_{1} .
$$

So we get

$$
\int_{0}^{T} e^{u(t)} d t \leqslant \frac{T \cdot \bar{r}_{1}}{b_{1}^{L}}
$$

Multiplying the first equation of (24) by $e^{u(t)}$ and integrating over $[0, T]$, we obtain

$$
\begin{aligned}
0 \leqslant \sum_{k=1}^{q} e^{u\left(t_{k}\right)} & {\left[1-\left(1+d_{1 k}\right)^{\lambda}\right] } \\
=\lambda \int_{0}^{T}\left[r_{1}(t) e^{u(t)}-b_{1}(t) e^{2 u(t)}\right. & \left.-\frac{c_{1}(t) e^{u(t)} e^{m v(t)}}{\alpha^{2}+\omega e^{u(t)}+\beta e^{2 u(t)}}\right] d t .
\end{aligned}
$$

From (28) and the integral mean value theorem, there exists a $\zeta_{1} \in[0, T]$ such that

$$
\begin{aligned}
\lambda \int_{0}^{T} & {\left[r_{1}(t) e^{u(t)}-b_{1}(t) e^{2 u(t)}\right] d t } \\
& -\sum_{k=1}^{q} e^{u\left(t_{k}\right)}\left[1-\left(1+d_{1 k}\right)^{\lambda}\right] \\
= & \int_{0}^{T} \frac{\lambda c_{1}(t) e^{u(t)} e^{m v(t)}}{\alpha^{2}+\omega e^{u(t)}+\beta e^{2 u(t)}} d t \\
= & c_{1}\left(\zeta_{1}\right) \int_{0}^{T} \frac{\lambda e^{u(t)} e^{m v(t)}}{\alpha^{2}+\omega e^{u(t)}+\beta e^{2 u(t)}} d t .
\end{aligned}
$$

Similarly, multiplying the second equation of (24) by $e^{v(\mathrm{t})}$ and integrating over $[0, T]$, we obtain

$$
\begin{aligned}
& 0 \leqslant \sum_{k=1}^{q} e^{v\left(t_{k}\right)}\left[1-\left(1+d_{2 k}\right)^{\lambda}\right] \\
& =\lambda \int_{0}^{T}\left[-r_{2}(t) e^{v(t)}-b_{2}(t) e^{2 v(t)}\right. \\
& \left.+\frac{c_{2}(t) e^{u(t)} e^{m v(t)}}{\alpha^{2}+\omega e^{u(t)}+\beta e^{2 u(t)}}\right] d t ;
\end{aligned}
$$

from (30) and the integral mean value theorem, there exists a $\zeta_{2} \in[0, T]$ such that

$$
\begin{aligned}
\lambda \int_{0}^{T}\left[r_{2}(t) e^{v(t)}+b_{2}(t) e^{2 v(t)}\right] d t \\
\quad+\sum_{k=1}^{q} e^{v\left(t_{k}\right)}\left[1-\left(1+d_{2 k}\right)^{\lambda}\right] \\
=\int_{0}^{T} \frac{\lambda c_{2}(t) e^{u(t)} e^{m v(t)}}{\alpha^{2}+\omega e^{u(t)}+\beta e^{2 u(t)}} d t \\
=c_{2}\left(\zeta_{2}\right) \int_{0}^{T} \frac{\lambda e^{u(t)} e^{m v(t)}}{\alpha^{2}+\omega e^{u(t)}+\beta e^{2 u(t)}} d t .
\end{aligned}
$$

From (29) and (31), we have

$$
\begin{gathered}
\lambda \int_{0}^{T}\left[r_{2}(t) e^{v(t)}+b_{2}(t) e^{2 v(t)}\right] d t \\
+\sum_{k=1}^{q} e^{v\left(t_{k}\right)}\left[1-\left(1+d_{2 k}\right)^{\lambda}\right] \\
=\frac{c_{2}\left(\zeta_{2}\right)}{c_{1}\left(\zeta_{1}\right)}\left[\lambda \int_{0}^{T}\left(r_{1}(t) e^{u(t)}-b_{1}(t) e^{2 u(t)}\right) d t\right. \\
\left.-\sum_{k=1}^{q} e^{u\left(t_{k}\right)}\left[1-\left(1+d_{1 k}\right)^{\lambda}\right]\right] .
\end{gathered}
$$

From (32), we get

$$
\int_{0}^{T} r_{2}(t) e^{v(t)} d t \leqslant \frac{c_{2}^{M}}{c_{1}^{L}} \int_{0}^{T} r_{1}(t) e^{u(t)} d t
$$


which yields

$$
r_{2}^{L} \int_{0}^{T} e^{v(t)} d t \leqslant \frac{c_{2}^{M} r_{1}^{M}}{c_{1}^{L}} \int_{0}^{T} e^{u(t)} d t
$$

From (27) and (34), we obtain

$$
\int_{0}^{T} e^{v(t)} d t \leqslant \frac{c_{2}^{M} r_{1}^{M} T \bar{r}_{1}}{c_{1}^{L} r_{2}^{L} b_{1}^{L}}
$$

Set

$$
A:=\max \left\{\frac{\bar{r}_{1}}{b_{1}^{L}}, \frac{c_{2}^{M} r_{1}^{M} \bar{r}_{1}}{c_{1}^{L} r_{2}^{L} b_{1}^{L}}\right\}
$$

and then, from (27), (35), and (36), we get

$$
\int_{0}^{T} e^{u(t)} d t \leqslant T \cdot A, \quad \int_{0}^{T} e^{\nu(t)} d t \leqslant T \cdot A
$$

Since $(u(t), v(t))^{\top} \in X$, there exist $\xi, \xi_{1}, \eta, \eta_{1} \in[0, T]$ such that

$$
\begin{array}{ll}
u(\xi)=\max _{t \in[0, T]} u(t), & u\left(\xi_{1}^{+}\right)=\min _{t \in[0, T]} u(t), \\
v(\eta)=\max _{t \in[0, T]} v(t), & v\left(\eta_{1}^{+}\right)=\min _{t \in[0, T]} v(t) .
\end{array}
$$

From (37) and (38), we see that

$$
u\left(\xi_{1}^{+}\right) \leqslant \ln A, \quad v\left(\eta_{1}^{+}\right) \leqslant \ln A .
$$

On the other hand, it follows from (24) that

$$
\begin{aligned}
\int_{0}^{T}|\dot{u}(t)| d t \leqslant & 2 \int_{0}^{T} r_{1}(t) d t \\
& +\sum_{k=1}^{q} \ln \left(1+d_{1 k}\right) \\
\leqslant & 2 \int_{0}^{T} r_{1}(t) d t=2 T \cdot \bar{r}_{1}, \\
\int_{0}^{T}|\dot{v}(t)| d t \leqslant & 2 \int_{0}^{T} r_{2}(t) d t \\
& +2 \int_{0}^{T} b_{2}(t) e^{v(t)} d t-\sum_{k=1}^{q} \ln \left(1+d_{2 k}\right) \\
\leqslant & 2 T \cdot \bar{r}_{2}+2 b_{2}^{M} T \cdot A \\
& -\sum_{k=1}^{q} \ln \left(1+d_{2 k}\right):=S_{1} .
\end{aligned}
$$

$$
\begin{aligned}
v(t) & \leqslant v\left(\eta_{1}^{+}\right)+\int_{0}^{T}|\dot{v}(s)| d s-2 \sum_{k=1}^{q} \ln \left(1+d_{2 k}\right) \\
& \leqslant \ln A+2 T \cdot \bar{r}_{2}+2 b_{2}^{M} T \cdot A-3 \sum_{k=1}^{q} \ln \left(1+d_{2 k}\right) \\
& :=S_{3} .
\end{aligned}
$$

Meanwhile, the first equation of (25) implies

$$
T \cdot \bar{r}_{1} \leqslant b_{1}^{M} \int_{0}^{T} e^{u(t)} d t+\frac{c_{1}^{M}}{\alpha^{2}} \int_{0}^{T} e^{m v(t)} d t-\sum_{k=1}^{q} \ln \left(1+d_{1 k}\right) .
$$

In view of $\int_{0}^{T} e^{m v(t)} d t \leqslant T^{1-m}\left(\int_{0}^{T} e^{\nu(t)} d t\right)^{m}$ and (34), we have from (44) that

$$
\begin{aligned}
T \cdot \bar{r}_{1} \leqslant & b_{1}^{M} \int_{0}^{T} e^{u(t)} d t \\
& +\frac{c_{1}^{M} T^{1-m}}{\alpha^{2}}\left(\frac{c_{2}^{M} r_{1}^{M}}{r_{2}^{L} c_{1}^{L}}\right)^{m}\left(\int_{0}^{T} e^{u(t)} d t\right)^{m} \\
& -\sum_{k=1}^{q} \ln \left(1+d_{1 k}\right) .
\end{aligned}
$$

If $0<\int_{0}^{T} e^{u(t)} d t<1$, then it follows from (45) that

$$
\begin{aligned}
\int_{0}^{T} e^{u(t)} d t & \geqslant\left(\frac{T \cdot \bar{r}_{1}+\sum_{k=1}^{q} \ln \left(1+d_{1 k}\right)}{b_{1}^{M}+\left(c_{1}^{M} T^{1-m} / \alpha^{2}\right)\left(c_{2}^{M} r_{1}^{M} / r_{2}^{L} c_{1}^{L}\right)^{m}}\right)^{1 / m} \\
& :=S_{4},
\end{aligned}
$$


so we have

$$
\int_{0}^{T} e^{u(t)} d t \geqslant \min \left\{1, S_{4}\right\}:=S_{5} .
$$

From (38) and (47), we have

$$
u(\xi) \geqslant \ln \frac{S_{5}}{T} .
$$

This, together with (40), leads to

$$
\begin{aligned}
u(t) & \geqslant u(\xi)-\int_{0}^{T}|\dot{u}(t)| d t+2 \sum_{k=1}^{q} \ln \left(1+d_{1 k}\right) \\
& \geqslant \ln \frac{S_{5}}{T}-2 T \cdot \bar{r}_{1}+2 \sum_{k=1}^{q} \ln \left(1+d_{1 k}\right) \\
& :=S_{6} .
\end{aligned}
$$

Let $H_{1}=\max \left\{\left|S_{2}\right|,\left|S_{6}\right|\right\}$; then from (42) and (49), we have

$$
\max _{t \in[0, T]}|u(t)| \leqslant H_{1}
$$

Let

$$
\begin{gathered}
\theta=\min \{x \in \\
\quad\{1,2, \ldots,\} \mid \frac{C_{2}^{L} S_{5} x}{\alpha^{2}+\omega e^{S_{2}}+\beta e^{2 S_{2}}}-\bar{r}_{2} T \\
\left.+\sum_{k=1}^{q} \ln \left(1+d_{2 k}\right)>0\right\} .
\end{gathered}
$$

Case 1. If $0<e^{(1-m) v(t)}<\theta^{-1}$, for any $t \in[0, T]$, then it follows from the second equation of (25) that

$$
\begin{aligned}
\bar{r}_{2} T & -\sum_{k=1}^{q} \ln \left(1+d_{2 k}\right)+\int_{0}^{T} b_{2}(t) e^{v(t)} d t \\
& =\int_{0}^{T} \frac{c_{2}(t) e^{u(t)}}{\alpha^{2}+\omega e^{u(t)}+\beta e^{2 u(t)}} e^{(m-1) v(t)} d t \\
& \geqslant \frac{C_{2}^{L} S_{5} \theta}{\alpha^{2}+\omega e^{S_{2}}+\beta e^{2 S_{2}}},
\end{aligned}
$$

and from (38) we get

$$
\begin{aligned}
& e^{v(\eta)} T \\
& \geqslant \int_{0}^{T} e^{v(t)} d t \\
& \geqslant \frac{\left[\left(C_{2}^{L} S_{5} \theta /\left(\alpha^{2}+\omega e^{S_{2}}+\beta e^{2 S_{2}}\right)\right)-\bar{r}_{2} T+\sum_{k=1}^{q} \ln \left(1+d_{2 k}\right)\right]}{b_{2}^{M}} \\
& :=S_{7},
\end{aligned}
$$

that is,

$$
v(\eta) \geqslant \ln \frac{S_{7}}{T}
$$

Similar to (49), from (41) and (54) we obtain

$$
\begin{aligned}
v(t) & \geqslant v(\eta)-\int_{0}^{T}|\dot{v}(t)| d t+2 \sum_{k=1}^{q} \ln \left(1+d_{2 k}\right) \\
& \geqslant \ln \frac{S_{7}}{T}-S_{1}+2 \sum_{k=1}^{q} \ln \left(1+d_{2 k}\right) \\
& :=S_{8} .
\end{aligned}
$$

Case 2. There exists $\gamma \in[0, T]$ such that $e^{(1-m) v(\gamma)} \geqslant \theta^{-1}$, that is,

$$
v(\gamma) \geqslant \frac{1}{m-1} \ln \theta .
$$

From (41) and (56) we obtain

$$
\begin{aligned}
v(t) & \geqslant v(\gamma)-\int_{0}^{T}|\dot{v}(t)| d t+2 \sum_{k=1}^{q} \ln \left(1+d_{2 k}\right) \\
& \geqslant \frac{1}{m-1} \ln \theta-S_{1}+2 \sum_{k=1}^{q} \ln \left(1+d_{2 k}\right) \\
& :=S_{9} .
\end{aligned}
$$

So we have

$$
v(t) \geqslant \min \left\{S_{8}, S_{9}\right\}:=S_{10} .
$$

Let $H_{2}=\max \left\{\left|S_{3}\right|,\left|S_{10}\right|\right\}$, then from (43) and (58), we get

$$
\max _{t \in[0, T]}|v(t)| \leqslant H_{2} .
$$

Clearly, $H_{1}$ and $H_{2}$ are independent of $\lambda$. Denote $H=H_{1}+$ $H_{2}+H_{0}$, where $H_{0}$ is taken sufficiently large such that each solution $\left(u^{*}, v^{*}\right)^{\top}$ (if the system has at least one solution) of the following system of algebraic equations:

$$
\begin{aligned}
& \bar{b}_{1} e^{u}-\bar{r}_{1}+\frac{\bar{c}_{1} e^{m v}}{\alpha^{2}+\omega e^{u}+\beta e^{2 u}}=\sum_{k=1}^{q} \ln \left(1+d_{1 k}\right), \\
& \bar{b}_{2} e^{v}+\bar{r}_{2}-\frac{\bar{c}_{2} e^{\alpha+(m-1) \beta}}{\alpha^{2}+\omega e^{u}+\beta e^{2 u}}=\sum_{k=1}^{q} \ln \left(1+d_{2 k}\right),
\end{aligned}
$$

satisfies $\left|u^{*}\right|+\left|v^{*}\right|<H_{0}$ and

$$
\max \left\{\left|S_{11}\right|,\left|S_{12}\right|\right\}+\max \left\{\left|S_{13}\right|,\left|S_{14}\right|\right\}<H_{0}
$$


where

$$
\begin{aligned}
& S_{11}=\ln \frac{\bar{r}_{1}}{\bar{b}_{1}} \\
& S_{12}=\min \left\{0, \ln \left(\frac{\bar{r}_{1}+\sum_{k=1}^{q} \ln \left(1+d_{1 k}\right)}{\bar{b}_{1}+\left(\bar{c}_{1} / \alpha^{2}\right)}\right)\right\} \text {, } \\
& S_{13}=\min \left\{0, \frac{1}{1-m}\right. \\
& \cdot \ln \left(\bar{c}_{2} e^{S_{12}}\right. \\
& \cdot\left(\left(\bar{b}_{2}+\bar{r}_{2}-\sum_{k=1}^{q} \ln \left(1+d_{2 k}\right)\right)\right. \\
& \left.\left.\left.\cdot\left(\alpha^{2}+\omega e^{S_{11}}+\beta e^{S_{11}}\right)\right)^{-1}\right)\right\} \\
& S_{14}=\frac{1}{m-1} \ln \left(-\frac{\bar{b}_{1} \alpha^{2} \sum_{k=1}^{q} \ln \left(1+d_{2 k}\right)}{\bar{c}_{2} \bar{r}_{1}}\right) \text {. }
\end{aligned}
$$

Let $\Omega=\{z \in X:\|z\|<H\}$; then $\Omega$ satisfies condition $\left[C_{1}\right]$ in Lemma 2. If $z \in \partial \Omega \cap \operatorname{Ker} L=\partial \Omega \cap \mathrm{R}^{2}$, then $z$ is a constant vector in $\mathrm{R}^{2}$ with $\|z\|=H$. So

QNz

$$
\begin{aligned}
& =Q N\left(\begin{array}{l}
u \\
v
\end{array}\right) \\
& =\left(\begin{array}{l}
\left(\bar{b}_{1} e^{u}-\bar{r}_{1}+\frac{\bar{c}_{1} e^{m v}}{\alpha^{2}+\omega e^{u}+\beta e^{2 u}}-\sum_{k=1}^{q} \ln \left(1+d_{1 k}\right)\right. \\
\bar{b}_{2} e^{v}+\bar{r}_{2}-\frac{\bar{c}_{2} e^{u+(m-1) v}}{\alpha^{2}+\omega e^{u}+\beta e^{2 u}}-\sum_{k=1}^{q} \ln \left(1+d_{2 k}\right)
\end{array}\right), \\
& \left.\left.\qquad\left(\begin{array}{l}
0 \\
0
\end{array}\right)\right\}_{k=1}^{q}\right) \neq 0,
\end{aligned}
$$

which shows that condition $\left[C_{2}\right]$ in Lemma 2 is satisfied. Finally, we prove that condition $\left[C_{3}\right]$ in Lemma 2 is satisfied. The isomorphism $J$ of $\operatorname{Im} Q$ onto Ker $L$ can be defined by

$$
\begin{aligned}
& J: \operatorname{Im} Q \longrightarrow \operatorname{Ker} L \\
& \left(\left(\begin{array}{l}
l_{1} \\
l_{2}
\end{array}\right),\left\{\left(\begin{array}{l}
0 \\
0
\end{array}\right)\right\}_{k=1}^{q}\right) \longmapsto\left(\begin{array}{l}
l_{1} \\
l_{2}
\end{array}\right) .
\end{aligned}
$$

For $z \in \Omega \cap \operatorname{Ker} L$, we have

$\mathrm{JQNz}$

$$
\begin{aligned}
& =\operatorname{JQN}\left(\begin{array}{l}
u \\
v
\end{array}\right) \\
& =\left(\begin{array}{c}
\bar{b}_{1} e^{u}-\bar{r}_{1}+\frac{\bar{c}_{1} e^{m v}}{\alpha^{2}+\omega e^{u}+\beta e^{2 u}}-\sum_{k=1}^{q} \ln \left(1+d_{1 k}\right) \\
\bar{b}_{2} e^{v}+\bar{r}_{2}-\frac{\bar{c}_{2} e^{u+(m-1) v}}{\alpha^{2}+\omega e^{u}+\beta e^{2 u}}-\sum_{k=1}^{q} \ln \left(1+d_{2 k}\right)
\end{array}\right) .
\end{aligned}
$$

Denote $\phi: \operatorname{Dom} L \times[0,1] \rightarrow X$ as the form

$$
\begin{aligned}
& \phi(u, v, \mu)=\left(\begin{array}{c}
\bar{b}_{1} e^{u}-\bar{r}_{1}-\sum_{k=1}^{q} \ln \left(1+d_{1 k}\right) \\
-\frac{\bar{c}_{2} e^{u+(m-1) v}}{\alpha^{2}+\omega e^{u}+\beta e^{2 u}}-\sum_{k=1}^{q} \ln \left(1+d_{2 k}\right)
\end{array}\right) \\
& +\mu\left(\begin{array}{c}
\frac{\bar{c}_{1} e^{m v}}{\alpha^{2}+\omega e^{u}+\beta e^{2 u}} \\
\bar{b}_{2} e^{v}+\bar{r}_{2}
\end{array}\right),
\end{aligned}
$$

where $\mu$ is a parameter. We will show that when $(u, v)^{\top} \in \partial \Omega \cap$ $\operatorname{Ker} L, \phi(u, v, \mu) \neq 0$ for any $\mu \in[0,1]$. Assume the conclusion is not true; that is, there is a constant vector $(u, v)^{\top}$ with $|u|+$ $|v|=H$ satisfying $\phi(u, v, \mu)=0$, that is,

$$
\begin{gathered}
\bar{b}_{1} e^{u}-\bar{r}_{1}-\sum_{k=1}^{q} \ln \left(1+d_{1 k}\right)+\frac{\mu \bar{c}_{1} e^{m v}}{\alpha^{2}+\omega e^{u}+\beta e^{2 u}}=0, \\
\mu \bar{b}_{2} e^{v}+\mu \bar{r}_{2}-\sum_{k=1}^{q} \ln \left(1+d_{2 k}\right)-\frac{\bar{c}_{2} e^{u+(m-1) v}}{\alpha^{2}+\omega e^{u}+\beta e^{2 u}}=0 .
\end{gathered}
$$

By (67) we easily see

$$
u \leqslant \ln \frac{\bar{r}_{1}}{\bar{b}_{1}}=S_{11},
$$

and we also get

$$
\begin{aligned}
\bar{r}_{1} & \leqslant \bar{b}_{1} e^{u}-\sum_{k=1}^{q} \ln \left(1+d_{1 k}\right)+\frac{\bar{c}_{1} e^{m v}}{\alpha^{2}+\omega e^{u}+\beta e^{2 u}} \\
& \leqslant \bar{b}_{1} e^{u}-\sum_{k=1}^{q} \ln \left(1+d_{1 k}\right)+\frac{\bar{c}_{1} e^{m v}}{\alpha^{2}} .
\end{aligned}
$$

Case 1. If $m v \leqslant u$, from (70) and $\left[D_{2}\right]$, we have $u \geqslant \ln \left(\left(\bar{r}_{1}+\right.\right.$ $\left.\left.\sum_{k=1}^{q} \ln \left(1+d_{1 k}\right)\right) /\left(\bar{b}_{1}+\left(\bar{c}_{1} / \alpha^{2}\right)\right)\right)$.

Case 2. If $m v>u$, there exists a $n^{*} \in N$, such that $u<m v<$ $n^{*} u$; from (70), we have

$$
\bar{r}_{1} \leqslant \bar{b}_{1} e^{u}-\sum_{k=1}^{q} \ln \left(1+d_{1 k}\right)+\frac{\bar{c}_{1} e^{n^{*} u}}{\alpha^{2}} .
$$


Case 2.1. If $0<e^{u}<1$, from (71) and [ $\left.D_{2}\right]$, we obtain $u \geqslant$ $\ln \left(\left(\bar{r}_{1}+\sum_{k=1}^{q} \ln \left(1+d_{1 k}\right)\right) /\left(\bar{b}_{1}+\left(\bar{c}_{1} / \alpha^{2}\right)\right)\right)$.

Case 2.2. If $e^{u} \geqslant 1$, we have $u \geqslant 0$.

So we have

$$
u \geqslant \min \left\{0, \ln \left(\frac{\bar{r}_{1}+\sum_{k=1}^{q} \ln \left(1+d_{1 k}\right)}{\bar{b}_{1}+\left(\bar{c}_{1} / \alpha^{2}\right)}\right)\right\}=S_{12} .
$$

Then from (69) and (72), we get $|u| \leqslant \max \left\{\left|S_{11}\right|,\left|S_{12}\right|\right\}$.

From (68), we have

$$
\frac{\bar{c}_{2} e^{u+(m-1) v}}{\alpha^{2}+\omega e^{u}+\beta e^{2 u}} \leqslant \bar{b}_{2} e^{v}+\bar{r}_{2}-\sum_{k=1}^{q} \ln \left(1+d_{2 k}\right)
$$

and from (69) and (72), we have

$$
\begin{aligned}
\frac{\bar{c}_{2} e^{S_{12}}}{\alpha^{2}+\omega e^{S_{11}}+\beta e^{2 S_{11}}} \leqslant & \bar{b}_{2} e^{(2-m) v} \\
& +\left(\bar{r}_{2}-\sum_{k=1}^{q} \ln \left(1+d_{2 k}\right)\right) e^{(1-m) v} .
\end{aligned}
$$

Case 1. If $v>0$, then we get the lower bounds of $v$.

Case 2. If $v<0$, then we see $e^{(1-m) v} \geqslant e^{(2-m) v}$, which together with (74) yields

$$
\frac{\bar{c}_{2} e^{S_{12}}}{\alpha^{2}+\omega e^{S_{11}}+\beta e^{S_{11}}} \leqslant\left(\bar{b}_{2}+\bar{r}_{2}-\sum_{k=1}^{q} \ln \left(1+d_{2 k}\right)\right) e^{(1-m) v},
$$

which implies

$$
\begin{aligned}
& v \geqslant \frac{1}{1-m} \\
& \cdot \ln \left(\bar{c}_{2} e^{S_{12}}\right. \\
& \\
& \quad\left(\left(\bar{b}_{2}+\bar{r}_{2}-\sum_{k=1}^{q} \ln \left(1+d_{2 k}\right)\right)\right. \\
& \left.\left.\cdot\left(\alpha^{2}+\omega e^{S_{11}}+\beta e^{S_{11}}\right)\right)^{-1}\right) .
\end{aligned}
$$

So we get

$$
\begin{aligned}
& v \geqslant \min \left\{0, \frac{1}{1-m}\right. \\
& \cdot \ln \left(\bar{c}_{2} e^{S_{12}}\right. \\
& \cdot\left(\left(\bar{b}_{2}+\bar{r}_{2}-\sum_{k=1}^{q} \ln \left(1+d_{2 k}\right)\right)\right. \\
& \left.\left.\left.\cdot\left(\alpha^{2}+\omega e^{S_{11}}+\beta e^{S_{11}}\right)\right)^{-1}\right)\right\} \\
& =S_{13} .
\end{aligned}
$$

From (68) and (69), we obtain

$$
\begin{aligned}
\frac{\bar{c}_{2} \bar{r}_{1} e^{(m-1) v}}{\bar{b}_{1} \alpha^{2}} & =\frac{\bar{c}_{2} e^{S_{11}} e^{(m-1) v}}{\alpha^{2}} \\
& \geqslant \frac{\bar{c}_{2} e^{u+(m-1) v}}{\alpha^{2}+\omega e^{u}+\beta e^{2 u}} \geqslant-\sum_{k=1}^{q} \ln \left(1+d_{2 k}\right),
\end{aligned}
$$

and then we have

$$
v \leqslant \frac{1}{m-1} \ln \left(-\frac{\bar{b}_{1} \alpha^{2} \sum_{k=1}^{q} \ln \left(1+d_{2 k}\right)}{\bar{c}_{2} \bar{r}_{1}}\right)=S_{14}
$$

Then from (77) and (79), we get $|v| \leqslant \max \left\{\left|S_{13}\right|,\left|S_{14}\right|\right\}$.

Therefore,

$$
|u|+|v| \leqslant \max \left\{\left|S_{11}\right|,\left|S_{12}\right|\right\}+\max \left\{\left|S_{13}\right|,\left|S_{14}\right|\right\}<H_{0}<H,
$$

which leads to a contradiction. Using the property of topological degree, we have

$$
\begin{aligned}
\operatorname{deg}\{ & \left.\operatorname{JQN}(u, v)^{\top}, \Omega \cap \operatorname{ker} L,(0,0)^{\top}\right\} \\
= & \operatorname{deg}\left\{\phi(u, v, 1), \Omega \cap \operatorname{ker} L,(0,0)^{\top}\right\} \\
= & \operatorname{deg}\left\{\phi(u, v, 0), \Omega \cap \operatorname{ker} L,(0,0)^{\top}\right\} .
\end{aligned}
$$

By $\left[D_{1}\right]$ and $\left[D_{2}\right]$, we see that the following system of algebraic equation

$$
\begin{gathered}
\bar{b}_{1} e^{u}-\bar{r}_{1}-\sum_{k=1}^{q} \ln \left(1+d_{1 k}\right)=0, \\
-\frac{\bar{c}_{2} e^{u+(m-1) v}}{k^{2}+\omega e^{u}+\beta e^{2 u}}-\sum_{k=1}^{q} \ln \left(1+d_{2 k}\right)=0,
\end{gathered}
$$

has a unique solution in $R^{2}$. Thus, a standard and direct calculation shows that

$$
\operatorname{deg}\left\{J Q N(u, v)^{\top}, \Omega \cap \operatorname{ker} L,(0,0)^{\top}\right\}=-1 .
$$


Obviously, the open set $\Omega$ satisfies all conditions in Lemma 2, and therefore we claim that system (9) has at least one $T$ periodic solution on $\bar{\Omega} \cap \operatorname{Dom} L$; that is, system (6) has at least one positive periodic solution. Thus we complete the proof.

Remark 4. Our model (6) is more general than those in $[24,25]$ since there are different types of Holling functions.
The results in $[24,25]$ do not give the decision on existence of positive solution to (6).

Remark 5. In our proof, by new tricks, we avoid the errors that existed in [23-25]. In the proof of Theorem 2.1 [23, page 230], the authors stated that: "let $L: \operatorname{Dom} L \subset X \rightarrow Y$, $u \rightarrow\left(u^{\prime}, \Delta u\left(t_{1}\right), \ldots, \Delta u\left(t_{q}\right)\right)$,

$$
N u=\left(\left[\begin{array}{c}
r_{1}(t)-D_{1}(t)-a_{11}(t) e^{u_{1}(t)}-a_{13}(t) e^{u_{3}(t)}+D_{1}(t) e^{u_{2}(t)-u_{1}(t)} \\
r_{2}(t)-D_{2}(t)-a_{22}(t) e^{u_{2}(t)}+D_{2}(t) e^{u_{1}(t)-u_{2}(t)} \\
-r_{3}(t)+a_{31}(t) e^{u_{1}\left(t-\tau_{1}\right)}-a_{33}(t) e^{u_{3}\left(t-\tau_{2}\right)}
\end{array}\right],\left\{\left[\begin{array}{l}
\ln \left(1+b_{1 k}\right) \\
\ln \left(1+b_{2 k}\right) \\
\ln \left(1+b_{3 k}\right)
\end{array}\right]\right\}_{k=1}^{q}\right)
$$

where

$$
\begin{gathered}
X=\left\{\left(u_{1}(t), u_{2}(t), u_{3}(t)\right)^{\top}\right. \\
\left.\in P C\left(\mathrm{R}, \mathrm{R}^{3}\right): u_{i}(t+T)=u_{i}(t), i=1,2,3\right\}, \\
Y=X \times \mathrm{R}^{3 q},
\end{gathered}
$$

$P C\left(\mathrm{R}, \mathrm{R}^{3}\right)=\left\{x: \mathrm{R} \rightarrow \mathrm{R}^{3} \mid x\right.$ be continuous at $t \neq t_{k}, x\left(t_{k}^{+}\right)$, $x\left(t_{k}^{-}\right)$exist and $\left.x\left(t_{k}^{-}\right)=x\left(t_{k}\right), k=1,2, \ldots,\right\}$." From this, we can derive that $u^{\prime} \in X$; that is, $u^{\prime}=\left(u_{1}^{\prime}(t), u_{2}^{\prime}(t), u_{3}^{\prime}(t)\right)^{\top} \in$ $P C\left(\mathrm{R}, \mathrm{R}^{3}\right)$ and $u_{i}^{\prime}(t+T)=u_{i}^{\prime}(t), i=1,2,3$. From the definition of $P C\left(\mathrm{R}, \mathrm{R}^{3}\right)$, we obtain that $\left(u_{1}^{\prime}\left(t_{k}\right), u_{2}^{\prime}\left(t_{k}\right), u_{3}^{\prime}\left(t_{k}\right)\right)$ exists, and then we can deduce that $u_{1}(t), u_{2}(t)$ and $u_{3}(t)$ are continuous at $t=t_{k}$. So we have $\Delta u\left(t_{1}\right)=0, \ldots, \Delta u\left(t_{q}\right)=0$ for any $u \in \operatorname{Dom} L$. So we get $L u \neq N u$, for any $u \in \operatorname{Dom} L$, which contradicts the conclusion of Theorem 2.1 [23]. Errors similarly reappear in proving Theorem 2.4 [24, page 3393] and Theorem 2.1 [25, page 1047].

\section{An Illustrative Example}

The following illustrative example demonstrates the effectiveness of our main result.

Example 1. Consider the following predator-prey system of Holling type IV function response with mutual interference and impulsive effects

$$
\begin{gathered}
\dot{x}(t)=x(t)((6+\sin t)-(2-\sin t) x(t)) \\
-\frac{(0.7+0.2 \sin t) x(t)}{9+2 x(t)+3 x^{2}(t)} y^{0.5}(t), \\
\dot{y}(t)=y(t)(-(0.03+0.02 \sin t)-(0.3-0.1 \sin t) y(t)) \\
+\frac{(0.3+0.5 \sin t) x(t)}{9+2 x(t)+3 x^{2}(t)} y^{0.5}(t), \\
\quad t \neq t_{k}, \quad k=1,2, \ldots,
\end{gathered}
$$

$$
\begin{aligned}
& \Delta x\left(t_{k}\right)=d_{1 k} x\left(t_{k}\right), \quad k=1,2, \ldots, \\
& \Delta y\left(t_{k}\right)=d_{2 k} y\left(t_{k}\right), \quad k=1,2, \ldots
\end{aligned}
$$

We fix the parameters $d_{1 k}=-0.5, d_{2 k}=-0.3, t_{k+3}=t_{k}+2 \pi$, $[0,2 \pi] \cap\left\{t_{k}\right\}=\left\{t_{1}, t_{2}, t_{3}\right\}$. By an easy calculation, we obtain $\bar{r}_{1}=6, \sum_{k=1}^{3} \ln \left(1+d_{1 k}\right)=-3 \ln 2$.

Therefore we have

$$
\begin{gathered}
\bar{r}_{1}=6>-3 \ln 2=\sum_{k=1}^{3} \ln \left(1+d_{1 k}\right), \\
T \bar{r}_{1}=12 \pi>-3 \ln 2=\sum_{k=1}^{3} \ln \left(1+d_{1 k}\right) .
\end{gathered}
$$

Thus, by Theorem 3 , system (86) has at least one positive $2 \pi$ periodic solution.

\section{Conclusion}

In this work, we have considered a more general predatorprey model with Holling type IV function response and the impulsive effect. By use of the continuation theorem in coincidence degree theory and new anaytical tricks, we have provided the sufficient conditions to ensure the existence of the positive solution to this model. We also point out minor errors in some papers on relevant models. In the future, investigation on the convergence of the positive solutions will be probably very interesting and significant since $(0,0)$ is obviously the solution of (6) and that zero solution stands for the extinction of the species.

\section{Conflict of Interests}

The authors declare that there is no conflict of interests regarding the publication of this paper.

\section{Acknowledgments}

The authors would like to thank the referee for his/her careful reading and kind suggestions. This research was supported 
by the National Natural Science Foundations of China (nos. 11171178 and 61104136) and the Science and Technology Project of High Schools of Shandong Province (no. J14LI09).

\section{References}

[1] L. L. Wang and W. T. Li, "Periodic solutions and permanence for a delayed nonautonomous ratio-dependent predator-prey model with Holling type functional response," Journal of Computational and Applied Mathematics, vol. 162, no. 2, pp. 341-357, 2004.

[2] S. H. Chen, J. H. Zhang, and T. Young, "Existence of positive periodic solution for nonautonomous predator-prey system with diffusion and time delay," Journal of Computational and Applied Mathematics, vol. 159, no. 2, pp. 375-386, 2003.

[3] R. Xu and L. S. Chen, "Persistence and stability for a twospecies ratio-dependent predator-prey system with time delay in a two-patch environment," Computers \& Mathematics with Applications, vol. 40, no. 4-5, pp. 577-588, 2000.

[4] R. Xu, M. A. J. Chaplain, and F. A. Davidson, "Periodic solution of a Lotka-Volterra predator-prey model with dispersion and time delays," Applied Mathematics and Computation, vol. 148, no. 2, pp. 537-560, 2004.

[5] X. Y. Song and L. S. Chen, "Persistence and global stability for nonautonomous predator-prey system with diffusion and time delay," Computers \& Mathematics with Applications, vol. 35, no. 6, pp. 33-40, 1998.

[6] M. Bohner, M. Fan, and J. M. Zhang, "Existence of periodic solutions in predator-prey and competition dynamic systems," Nonlinear Analysis: Real World Applications, vol. 7, no. 5, pp. 1193-1204, 2006.

[7] J. A. Cui, "Dispersal permanence of a periodic predatorprey system with Beddington-DeAngelis functional response," Nonlinear Analysis: Theory, Methods \& Application, vol. 64, no. 3, pp. 440-456, 2006.

[8] M. Hassell, "Density dependence in single-species population," Journal of Animal Ecology, vol. 44, pp. 283-295, 1975.

[9] K. Wang, "Permanence and global asymptotical stability of a predator-prey model with mutual interference," Nonlinear Analysis: Real World Applications, vol. 12, no. 2, pp. 1062-1071, 2011.

[10] K. Wang, "Existence and global asymptotic stability of positive periodic solution for a predator-prey system with mutual interference," Nonlinear Analysis: Real World Applications, vol. 10, no. 5, pp. 2774-2783, 2009.

[11] X. Lin and F. D. Chen, "Almost periodic solution for a Volterra model with mutual interference and Beddington-DeAngelis functional response," Applied Mathematics and Computation, vol. 214, no. 2, pp. 548-556, 2009.

[12] Y. S. Lv and Z. J. Du, "Existence and global attractivity of a positive periodic solution to a Lotka-Volterra model with mutual interference and Holling III type functional response," Nonlinear Analysis: Real World Applications, vol. 12, no. 6, pp. 3654-3664, 2011.

[13] K. Wang and Y. L. Zhu, "Global attractivity of positive periodic solution for a Volterra model," Applied Mathematics and Computation, vol. 203, no. 2, pp. 493-501, 2008.

[14] X. L. Wang, Z. J. Du, and J. Liang, "Existence and global attractivity of positive periodic solution to a Lotka-Volterra model," Nonlinear Analysis: Real World Applications, vol. 11, no. 5, pp. 4054-4061, 2010.
[15] J. F. Andrews, "A mathematical model for the continuous culture of microorganisms utilizing inhibitory substrates," Biotechnology and Bioengineering, vol. 10, no. 6, pp. 707-723, 1968.

[16] D. D. Bainov and P. S. Simeonov, Impulsive Differential Equations: Periodic Solutions and Applications, Longman Scientific \& Technical, New York, NY, USA, 1993.

[17] X. Q. Wang, W. M. Wang, and X. L. Lin, "Dynamics of a twoprey one-predator system with Watt-type functional response and impulsive control strategy," Chaos, Solitons and Fractals, vol. 40, no. 5, pp. 2392-2404, 2009.

[18] X. Wang, W. Wang, and X. Lin, "Dynamics of a periodic Watt-type predator-prey system with impulsive effect," Chaos, Solitons and Fractals, vol. 39, no. 3, pp. 1270-1282, 2009.

[19] X. Q. Wang, W. M. Wang, and X. L. Lin, "Chaotic behavior of a Watt-type predator-prey system with impulsive control strategy," Chaos, Solitons and Fractals, vol. 37, no. 3, pp. 706-718, 2008.

[20] X. N. Liu and L. S. Chen, "Global dynamics of the periodic logistic system with periodic impulsive perturbations," Journal of Mathematical Analysis and Applications, vol. 289, no. 1, pp. 279-291, 2004.

[21] H. Baek, "Species extinction and permanence of an impulsively controlled two-prey one-predator system with seasonal effects," Biosystems, vol. 98, no. 1, pp. 7-18, 2009.

[22] M. X. He and F. D. Chen, "Dynamic behaviors of the impulsive periodic multi-species predator-prey system," Computers \& Mathematics with Applications, vol. 57, no. 2, pp. 248-265, 2009.

[23] J. H. Shen and J. L. Li, "Existence and global attractivity of positive periodic solutions for impulsive predator-prey model with dispersion and time delays," Nonlinear Analysis: Real World Applications, vol. 10, no. 1, pp. 227-243, 2009.

[24] Z. G. Luo, B. X. Dai, and Q. H. Zhang, "Existence of positive periodic solutions for an impulsive semi-ratio-dependent predator-prey model with dispersion and time delays," Applied Mathematics and Computation, vol. 215, no. 9, pp. 3390-3398, 2010.

[25] K. Wang and Y. L. Zhu, "Periodic solutions, permanence and global attractivity of a delayed impulsive prey-predator system with mutual interference," Nonlinear Analysis: Real World Applications, vol. 14, no. 2, pp. 1044-1054, 2013.

[26] X. Q. Yuan, Y. H. Xia, and D. O'Regan, "Nonautonomous impulsive systems with unbounded nonlinear terms," Applied Mathematics and Computation, vol. 245, pp. 391-403, 2014.

[27] Y. H. Xia, X. D. Chen, and V. G. Romanovski, "On the linearization theorem of fenner and pinto," Journal of Mathematical Analysis and Applications, vol. 400, no. 2, pp. 439-451, 2013.

[28] R. E. Gaines and J. L. Mawhin, Coincidence Degree and Nonlinear Differential Equations, Springer, Berlin, Germany, 1977. 


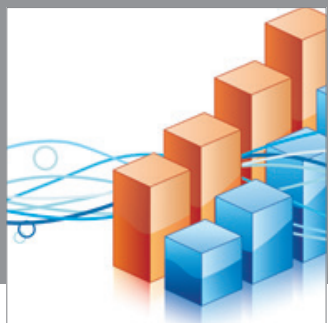

Advances in

Operations Research

mansans

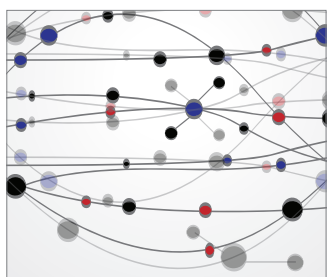

The Scientific World Journal
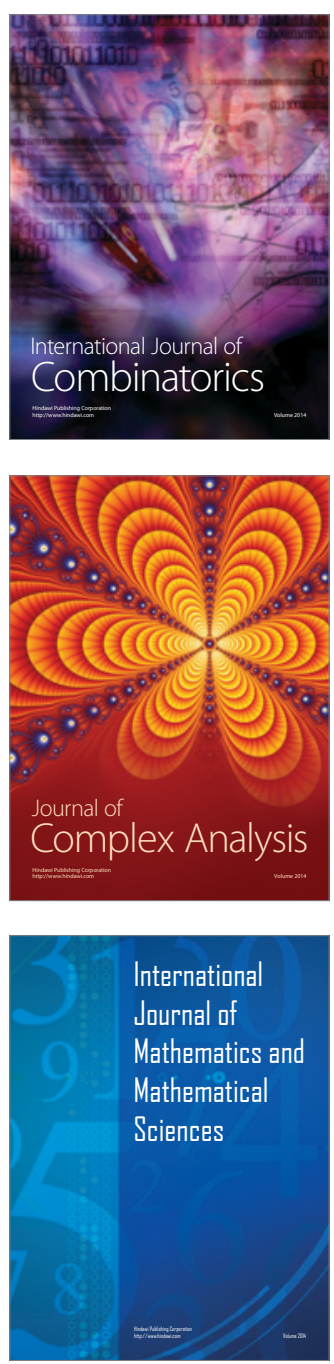
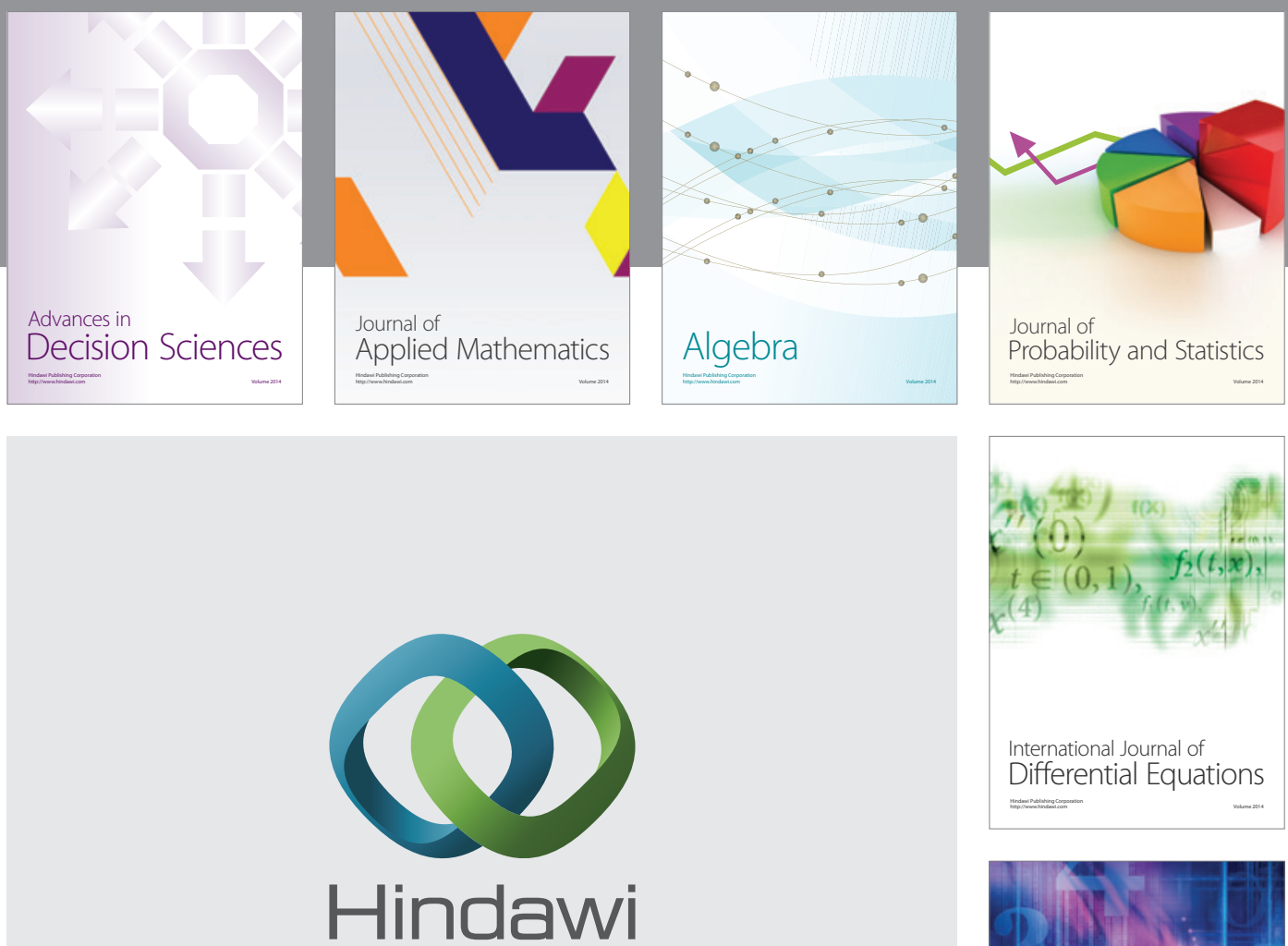

Submit your manuscripts at http://www.hindawi.com
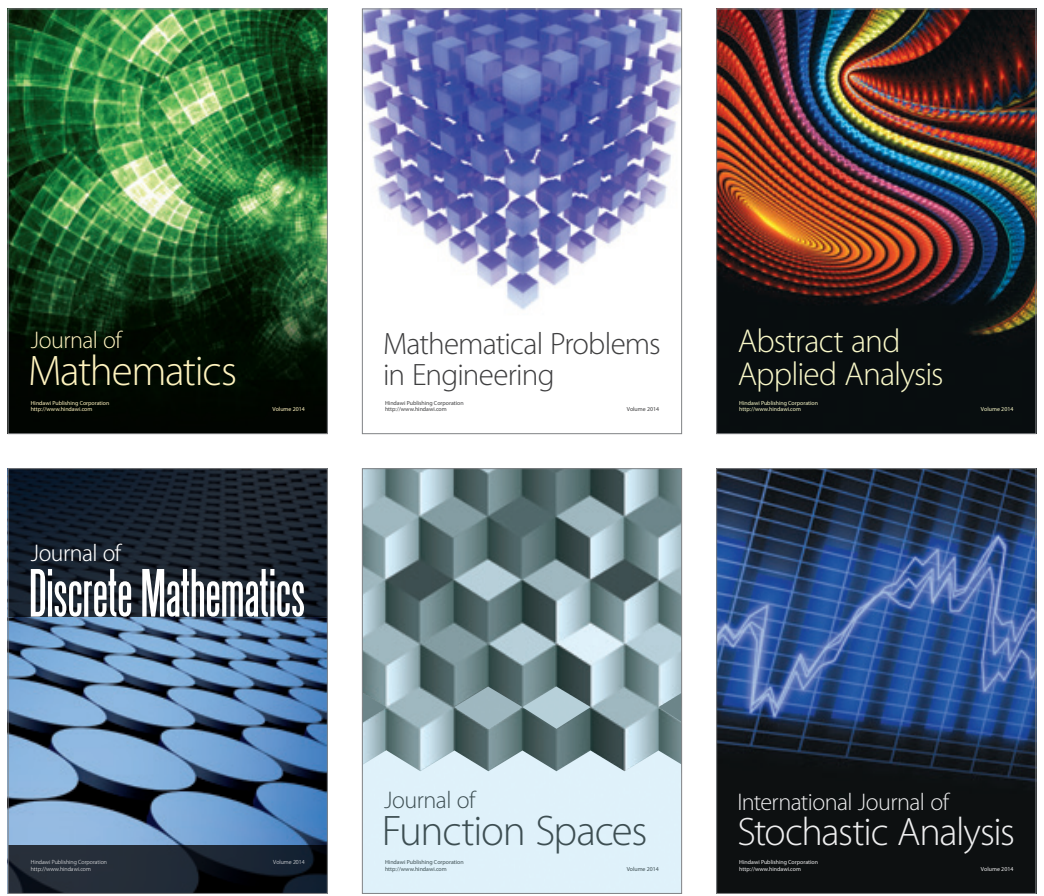

Journal of

Function Spaces

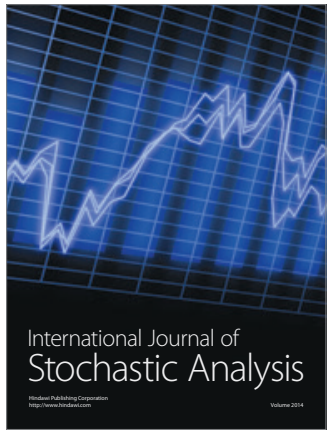

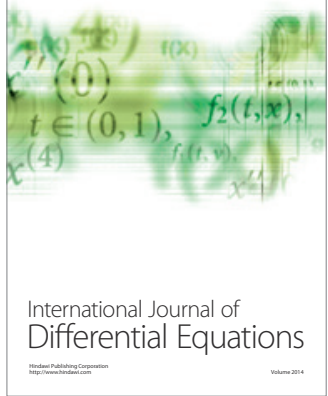
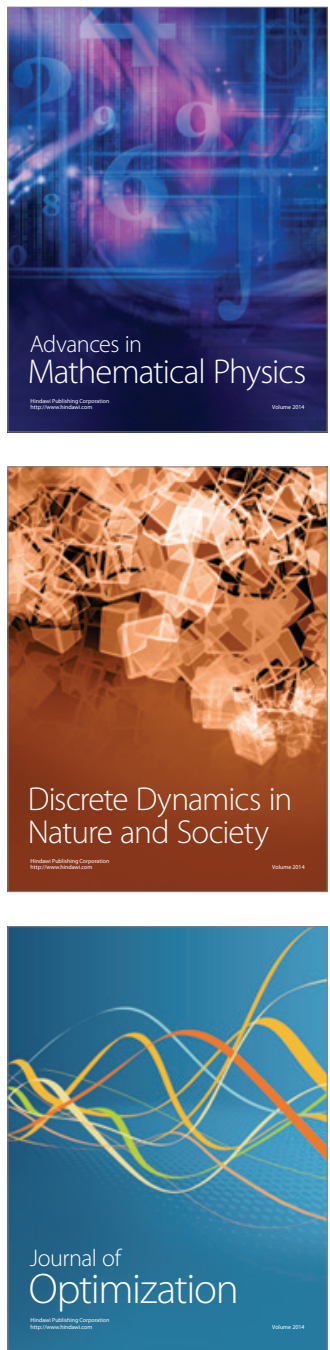